A highly accurate study of a helium atom under pressure

This article has been downloaded from IOPscience. Please scroll down to see the full text article.

2009 J. Phys. A: Math. Theor. 42265004

(http://iopscience.iop.org/1751-8121/42/26/265004)

The Table of Contents and more related content is available

Download details:

IP Address: 140.112.113.225

The article was downloaded on 02/07/2009 at 08:37

Please note that terms and conditions apply. 


\title{
A highly accurate study of a helium atom under pressure
}

\author{
Cecil Laughlin ${ }^{1,2}$ and Shih-I Chu ${ }^{2,3}$ \\ ${ }^{1}$ School of Mathematical Sciences, University of Nottingham, Nottingham NG7 2RD, UK \\ ${ }^{2}$ Center for Quantum Science and Engineering, National Taiwan University, Taipei, Taiwan \\ ${ }^{3}$ Department of Chemistry, University of Kansas, Lawrence, KS 66045, USA \\ E-mail: Cecil.Laughlin@nottingham.ac.uk
}

Received 19 March 2009, in final form 18 May 2009

Published 16 June 2009

Online at stacks.iop.org/JPhysA/42/265004

\begin{abstract}
Basis sets incorporating the interelectronic distance $r_{12}$ are employed in variational calculations of the non-relativistic ground-state energies and pressures of a helium atom confined at the centre of an impenetrable spherical cavity and results are presented for a series of cavity radii $R_{\mathrm{c}}$. The calculated values asymptotically approach the correct values in the limits $R_{\mathrm{c}} \rightarrow 0$ and $R_{\mathrm{c}} \rightarrow \infty$, and a simple analytical expression is obtained which makes accurate predictions for small cavities. The same method is also used to study two penetrable cavities modelled by Gaussian and harmonic potential wells. It is concluded that the present results are the most accurate currently available for a confined helium atom. The calculation method employs Gaussian quadrature to evaluate integrals and can very easily be adapted to any spherical confining potential.
\end{abstract}

PACS numbers: $31.15 .-\mathrm{p}, 31.15 . \mathrm{Ar}, 31.25 \mathrm{~Eb}$

\section{Introduction}

There have been many studies of confined atomic systems over the years, starting with the early pioneering work of Michels et al [1] more than 70 years ago, which was further developed by Sommerfeld and Welker [2] and by De Groot and Ten Seldam [3]. Of particular interest was the effects of high pressure on a compressed hydrogen atom and the ground-state polarizability was estimated for a series of confinement radii [1]. Since then many investigations have been undertaken and the compressed hydrogen atom is now well understood when enclosed in either impenetrable or penetrable cavities of both spherical and non-spherical shape; we do not give an extensive list here but refer the reader to recent comprehensive reviews [4-9]. An atom enclosed in an impenetrable cavity is, of course, a highly idealized model and its limitations 
were clearly recognized [3]. However, as pointed out in Fowler's extensive work on 1-electron problems [10], it does serve as a framework for discussing the properties of localized systems.

Helium, and other, atoms have, by contrast, received much less attention. As helium plays a central rôle in the study of multi-electron systems it is thus desirable to understand its behaviour under compression, particularly as the unconfined atom is now comprehensively documented to extremely high accuracy $[11,12]$. In the current paper we therefore implement a high-accurate study of the ground-state energy levels of a helium atom confined at the centre of a spherical cavity, of finite radius $R_{\mathrm{c}}$, and thus determine the energies of the atom under pressure. As will be seen the results obtained are the most reliable yet achieved for this system. The current implementation of the approach used here allows penetrable potentials to be incorporated in a straightforward manner; such potentials are thought to be desirable in practice to model confined systems [13] and we therefore also present some results for penetrable potentials.

\section{The non-relativistic Schrödinger equation for a confined helium-like atom}

We consider the non-relativistic Schrödinger equation

$$
\left[-\frac{1}{2} \nabla_{1}^{2}-\frac{1}{2} \nabla_{2}^{2}-\frac{Z}{r_{1}}-\frac{Z}{r_{2}}+\frac{1}{r_{12}}+V_{\mathrm{c}}\left(r_{1}, r_{2}\right)\right] \Psi\left(\mathbf{r}_{1}, \mathbf{r}_{2}\right)=E \Psi\left(\mathbf{r}_{1}, \mathbf{r}_{2}\right),
$$

for a helium-like atom, with nuclear charge $Z$, enclosed in a spherical cavity, of radius $R_{\mathrm{c}}$. The nucleus is assumed fixed at the centre of the cavity and we note that, for finite $R_{\mathrm{c}}$, it is not therefore possible to separate out the translational motion of the centre of mass of the system. $V_{\mathrm{c}}\left(r_{1}, r_{2}\right)$ is a confinement potential used to model the effects of pressure on the atom. For example, if the atom is enclosed within an impenetrable spherical cavity then

$$
V_{\mathrm{c}}\left(r_{1}, r_{2}\right)=\left\{\begin{array}{l}
0, \quad 0 \leqslant r_{1}, r_{2}<R_{\mathrm{c}}, \\
\infty, \quad r_{1} \geqslant R_{\mathrm{c}} \text { or } \quad r_{2} \geqslant R_{\mathrm{c}}
\end{array}\right.
$$

and the boundary conditions would require $\Psi\left(\mathbf{r}_{1}, \mathbf{r}_{2}\right)$ to vanish when $r_{1}=R_{\mathrm{c}}$ or when $r_{2}=R_{\mathrm{c}}$. Other types of penetrable cavities are possible; for example, Xie [14] considered spherical Gaussian potential wells, whereas Sako and Diercksen $[15,16]$ considered various harmonic constraining forms, both spherical and non-spherical, in their studies of the hydrogen negative ion, the helium atom and 2-electron quantum dots. The main interest of the latter authors was to investigate the spectra, density distribution and character of the three systems.

In the present paper we concentrate on helium enclosed within an impenetrable spherical box but also consider briefly some other penetrable boxes. The methods developed are quite general and may be used to investigate the properties of any spherically confined 2-electron system.

The eigenfunctions $\Psi\left(\mathbf{r}_{1}, \mathbf{r}_{2}\right)$ and associated eigenenergies $E$ are, of course, functions of $R_{\mathrm{c}}$ but we do not usually indicate this dependence explicitly (on occasions where necessary we add the superscript $\left(R_{\mathrm{c}}\right)$, or $(\infty)$, where $(\infty)$ indicates the unconfined atom).

\section{Method}

We use a variational approach to solve the Schrödinger equation (1). In order to satisfy the Dirichlet boundary conditions that $\Psi$ vanish on the surface of the impenetrable confining sphere we need to ensure the trial functions vanish there too. An easy way to do this is to introduce cut-off functions

$$
W_{\mathrm{c}}\left(r_{1}, r_{2}\right)=w_{\mathrm{c}}\left(r_{1}\right) w_{\mathrm{c}}\left(r_{2}\right),
$$


where $w_{\mathrm{c}}\left(R_{\mathrm{c}}\right)=0$. Several forms of $w_{\mathrm{c}}(r)$ have been proposed and used, including the linear form $w_{\mathrm{c}}^{(1)}(r)=R_{\mathrm{c}}-r[17,18]$ and the quadratic form $w_{\mathrm{c}}^{(2)}(r)=R_{\mathrm{c}}^{2}-r^{2}$ [19]. As well as $w_{\mathrm{c}}^{(1)}(r)$ we have also, in this work, employed the following non-linear form of the cut-off function:

$$
w_{\mathrm{c}}^{(3)}(r)=\left(1-\frac{r}{R_{\mathrm{c}}}\right) \exp \left(-\frac{r}{R_{\mathrm{c}}}\right) .
$$

We note that $w_{\mathrm{c}}^{(3)}(r)$ has the desirable property that $w_{\mathrm{c}}^{(3)^{\prime}}(0)=0$ so, unlike $w_{\mathrm{c}}^{(1)}(r)$, it does not have a cusp at the origin. This feature leads to more rapid convergence of the variational energies as the trial wavefunction becomes more flexible [19] and so $w_{\mathrm{c}}^{(3)}(r)$ provides more accurate lower bounds for ground-state energies than $w_{\mathrm{c}}^{(1)}(r)$, especially for smaller basis set expansions. Gorecki and Byers Brown [20] have described an iterative perturbation method to determine an optimum cut-off function $w_{\mathrm{c}}(r)$ when the unconfined-atom wavefunction is known. However, in our approach, $w_{\mathrm{c}}(r)$ is specified and the wavefunction is variationally optimized.

To give a precise description of electron correlation effects, highly accurate calculations for 2-electron atomic ions generally require the use of variational trial wavefunctions which depend explicitly on $r_{12}$, the electron-electron separation. Hylleraas-type functions of the form

$$
\Psi\left(\mathbf{r}_{1}, \mathbf{r}_{2}\right)=A_{12} \sum_{n m k} C_{n m k} r_{1}^{n} r_{2}^{m} r_{12}^{k} \mathrm{e}^{-\alpha r_{1}-\beta r_{2}-\gamma r_{12}} Y_{l_{1} l_{2} L}\left(\hat{\mathbf{r}}_{1}, \hat{\mathbf{r}}_{2}\right)
$$

where $A_{12}$ is an anti-symmetrization operator and $Y_{l_{1} l_{2} L}\left(\hat{\mathbf{r}}_{1}, \hat{\mathbf{r}}_{2}\right)$ denotes a vector-coupled product of spherical harmonics of total angular momentum $L$, have long been used. In practice, the calculations are usually performed using perimetric coordinates [21]. A difficulty with the direct application of a variational method using trial functions (5) is that the terms in the expansion effectively lose their linear independence as $n, m$ and $k$ are increased to make $\Psi$ more flexible. To overcome this problem we have, instead, constructed symmetrized trial functions of the form

$$
\begin{array}{r}
\Psi\left(\mathbf{r}_{1}, \mathbf{r}_{2}\right)=\sum_{n m k} \sum_{\nu} C_{n m k}^{(v)} N_{n m k}^{(v)}\left[\psi_{n m k}^{(v)}\left(r_{1}, r_{2}, r_{12}\right) Y_{l_{1} l_{2} L}\left(\hat{\mathbf{r}}_{1}, \hat{\mathbf{r}}_{2}\right)\right. \\
\left.+(-1)^{S+L+l_{1}+l_{2}} \psi_{n m k}^{(v)}\left(r_{2}, r_{1}, r_{12}\right) Y_{l_{2} l_{1} L}\left(\hat{\mathbf{r}}_{1}, \hat{\mathbf{r}}_{2}\right)\right]
\end{array}
$$

In (6) $N_{n m k}^{(\nu)}$ is a normalization factor,

$$
\psi_{n m k}^{(v)}\left(r_{1}, r_{2}, r_{12}\right)=\frac{1}{r_{1}} \phi_{n \lambda}\left(\alpha_{\nu} r_{1}\right) \frac{1}{r_{2}} \phi_{m \mu}\left(\beta_{\nu} r_{2}\right) g_{k}\left(\gamma_{\nu} r_{12}\right),
$$

where the normalized $\phi_{n \lambda}\left(r_{1}\right)$ is defined in terms of the generalized Laguerre polynomial $L_{n}^{(\lambda)}\left(r_{1}\right)[22]$ by

$$
\phi_{n \lambda}\left(r_{1}\right)=\left(\frac{n !}{(n+\lambda) !}\right)^{\frac{1}{2}} r_{1}^{\frac{1}{2} \lambda} L_{n}^{(\lambda)}\left(r_{1}\right) \mathrm{e}^{-\frac{1}{2} r_{1}},
$$

with $\lambda=2 l_{1}+2,\left(\mu=2 l_{2}+2\right)$ and $g_{k}$ has the form

$$
g_{k}\left(\gamma r_{12}\right)=r_{12}^{k} \mathrm{e}^{-\gamma r_{12}} \text {. }
$$

The index $v$ labels the values of the nonlinear parameters $\alpha_{\nu}, \beta_{v}$ and $\gamma_{v}$ in the trial functions. For fixed $\alpha_{\nu},\left\{\phi_{n \lambda}\left(\alpha_{\nu} r\right)\right\}_{n=0}^{n=\infty}$ is a complete orthonormal set of 1-electron functions, so trial functions of the form (6) suffer much less from problems of linear dependence than do functions of the form (5), as recognized by Pekeris [21]. 
For an unconfined atom the Hamiltonian and overlap matrix elements needed in the variational calculations can be evaluated using the angular-momentum re-coupling techniques introduced by Drake [23] for the angular variables and numerical Gauss-Laguerre quadrature for the radial variables. For example, consider the overlap matrix element $S_{12}=\left\langle\Psi_{1}\left(\mathbf{r}_{1}, \mathbf{r}_{2}\right) \mid \Psi_{2}\left(\mathbf{r}_{1}, \mathbf{r}_{2}\right)\right\rangle$, where $\Psi_{1}$ and $\Psi_{2}$ are functions as specified by equation (6). $S_{12}$ can be written as a sum of terms of the form

$$
\begin{aligned}
2 N_{1} N_{2}\left\langle\psi _ { 1 } \left( r_{1},\right.\right. & \left.r_{2}, r_{12}\right) Y_{l_{1} l_{2} L}\left(\hat{\mathbf{r}}_{1}, \hat{\mathbf{r}}_{2}\right) \\
& +(-1)^{S+L+l_{1}+l_{2}} \psi_{1}\left(r_{2}, r_{1}, r_{12}\right) Y_{l_{2} l_{1} L}\left(\hat{\mathbf{r}}_{1}, \hat{\mathbf{r}}_{2}\right)\left|\psi_{2}\left(r_{1}, r_{2}, r_{12}\right) Y_{l_{1}^{\prime} l_{2}^{\prime} L}\left(\hat{\mathbf{r}}_{1}, \hat{\mathbf{r}}_{2}\right)\right\rangle \\
= & 2 N_{1} N_{2}\left[\sum_{\Lambda_{1}} C_{\Lambda_{1}} I_{\Lambda_{1}}\left(\psi_{1}\left(r_{1}, r_{2}, r_{12}\right) \psi_{2}\left(r_{1}, r_{2}, r_{12}\right)\right)\right. \\
& \left.+(-1)^{S+L+l_{1}+l_{2}} \sum_{\Lambda_{2}} C_{\Lambda_{2}} I_{\Lambda_{2}}\left(\psi_{1}\left(r_{2}, r_{1}, r_{12}\right) \psi_{2}\left(r_{1}, r_{2}, r_{12}\right)\right)\right]
\end{aligned}
$$

where

$$
\begin{aligned}
I_{\Lambda}(F) & =\frac{1}{2} \int_{0}^{\infty} r_{1} \mathrm{~d} r_{1} \int_{0}^{\infty} r_{2} \mathrm{~d} r_{2} \int_{\left|r_{1}-r_{2}\right|}^{r_{1}+r_{2}} F\left(r_{1}, r_{2}, r_{12}\right) P_{\Lambda}\left(\cos \theta_{12}\right) r_{12} \mathrm{~d} r_{12} \\
& =\frac{1}{8} \int_{0}^{\infty} \int_{0}^{\infty} \int_{0}^{\infty} r_{1} r_{2} r_{12} F\left(r_{1}, r_{2}, r_{12}\right) P_{\Lambda}\left(\cos \theta_{12}\right) \mathrm{d} x \mathrm{~d} y \mathrm{~d} z
\end{aligned}
$$

with

$$
r_{1}=\frac{1}{2}(x+z), \quad r_{2}=\frac{1}{2}(y+x), \quad r_{12}=\frac{1}{2}(z+y)
$$

and

$$
\cos \theta_{12}=\frac{r_{1}^{2}+r_{2}^{2}-r_{12}^{2}}{2 r_{1} r_{2}} .
$$

The angular coefficients $C_{\Lambda}$ have been specified by Drake [23].

When expressed in the form (12) the integrals $I_{\Lambda_{1}}\left(\psi_{1} \psi_{2}\right)$ and $I_{\Lambda_{2}}\left(\psi_{1} \psi_{2}\right)$ can easily be evaluated exactly by employing Gauss-Laguerre integration. Gauss-Laguerre quadrature is also employed when the atom is confined by a penetrable potential (since the upper limits in the triple integral (12) still remain infinite) but it is not suitable for the impenetrable case. We then use equation (11), with the $r_{1}$ and $r_{2}$ upper integration infinite limits replaced by $R_{\mathrm{c}}$, and employ Gauss-Legendre quadrature instead.

The matrix elements $\left\langle\Psi_{1}\left(\mathbf{r}_{1}, \mathbf{r}_{2}\right)\left|-\frac{Z}{r_{1}}-\frac{Z}{r_{2}}+\frac{1}{r_{12}}\right| \Psi_{2}\left(\mathbf{r}_{1}, \mathbf{r}_{2}\right)\right\rangle$ can be evaluated in an identical fashion. The calculation of $\left\langle\Psi_{1}\left(\mathbf{r}_{1}, \mathbf{r}_{2}\right)\left|-\frac{1}{2} \nabla_{1}^{2}-\frac{1}{2} \nabla_{2}^{2}\right| \Psi_{2}\left(\mathbf{r}_{1}, \mathbf{r}_{2}\right)\right\rangle$ proceeds via the expression

$$
\begin{aligned}
& \nabla_{1}^{2} \frac{1}{r_{1}} \phi_{n \lambda}\left(\alpha r_{1}\right) g_{k}\left(\gamma r_{12}\right) Y_{l_{1} l_{2}}\left(\hat{\mathbf{r}}_{1}, \hat{\mathbf{r}}_{2}\right) \\
&=\frac{1}{r_{1}}\left[\frac{\alpha^{2}}{4}-\frac{\alpha\left(n+\frac{\lambda}{2}\right)}{r_{1}}-\frac{n}{r_{1}^{2}}+\frac{k(k+1)}{r_{12}^{2}}-\frac{2 \gamma(k+1)}{r_{12}}+\gamma^{2}\right. \\
&\left.+\frac{r_{1}^{2}-r_{2}^{2}+r_{12}^{2}}{r_{1} r_{12}}\left(\frac{n+\frac{\lambda}{2}-1}{r_{1}}-\frac{\alpha}{2}\right)\left(\frac{k}{r_{12}}-\gamma\right)\right] \phi_{n \lambda}\left(\alpha r_{1}\right) g_{k}\left(\gamma r_{12}\right) Y_{l_{1} l_{2} L}\left(\hat{\mathbf{r}}_{1}, \hat{\mathbf{r}}_{2}\right) \\
&+\frac{\sqrt{n(n+\lambda)}}{r_{1}^{2}}\left[\frac{1}{r_{1}}-\frac{r_{1}^{2}-r_{2}^{2}+r_{12}^{2}}{r_{1} r_{12}}\left(\frac{k}{r_{12}}-\gamma\right)\right] \phi_{n-1, \lambda}\left(\alpha r_{1}\right) g_{k}\left(\gamma r_{12}\right) Y_{l_{1} l_{2} L}\left(\hat{\mathbf{r}}_{1}, \hat{\mathbf{r}}_{2}\right) \\
&-\frac{2 r_{2}}{r_{1}^{2} r_{12}}\left(\frac{k}{r_{12}}-\gamma\right) \phi_{n \lambda}\left(\alpha r_{1}\right) g_{k}\left(\gamma r_{12}\right) \sum_{k_{1} k_{2}} W_{k_{1} k_{2}}\left(l_{1} l_{2} L\right) Y_{k_{1} k_{2} L}\left(\hat{\mathbf{r}}_{1}, \hat{\mathbf{r}}_{2}\right) .
\end{aligned}
$$


The algebraic coefficients $W_{k_{1} k_{2}}\left(l_{1} l_{2} L\right)$ in (15) are given by

$$
W_{k_{1} k_{2}}\left(l_{1} l_{2} L\right)=(-1)^{l_{1}+k_{2}+L}\left\{\begin{array}{ccc}
L & k_{2} & k_{1} \\
1 & l_{1} & l_{2}
\end{array}\right\}\left\langle k_{1}\|r \nabla\| l_{1}\right\rangle\left\langle k_{2}\|\hat{\mathbf{r}}\| l_{2}\right\rangle,
$$

where

$$
\langle k\|r \nabla\| l\rangle=\left\{\begin{array}{lll}
-l \sqrt{l+1} & \text { if } & k=l+1 \\
-(l+1) \sqrt{l} & \text { if } & k=l-1
\end{array}\right.
$$

and

$$
\langle k\|\hat{\mathbf{r}}\| l\rangle=\left\{\begin{array}{lll}
\sqrt{l+1} & \text { if } & k=l+1 \\
-\sqrt{l} & \text { if } & k=l-1
\end{array}\right.
$$

When a cut-off function $w_{c}\left(r_{1}\right)$ is introduced we have the result

$$
\begin{aligned}
\nabla_{1}^{2} \frac{1}{r_{1}} \phi_{n \lambda}\left(\alpha r_{1}\right) & g_{k}\left(\gamma r_{12}\right) w_{c}\left(r_{1}\right) Y_{l_{1} l_{2} L}\left(\hat{\mathbf{r}}_{1}, \hat{\mathbf{r}}_{2}\right)=w_{c}\left(r_{1}\right) \nabla_{1}^{2} \frac{1}{r_{1}} \phi_{n \lambda}\left(\alpha r_{1}\right) g_{k}\left(\gamma r_{12}\right) Y_{l_{1} l_{2} L}\left(\hat{\mathbf{r}}_{1}, \hat{\mathbf{r}}_{2}\right) \\
& +w_{c}^{\prime}\left(r_{1}\right)\left[2\left(\frac{1}{r_{1}} \phi_{n \lambda}\left(\alpha r_{1}\right)\right)^{\prime} g_{k}\left(\gamma r_{12}\right)\right. \\
& \left.+\frac{r_{1}^{2}-r_{2}^{2}+r_{12}^{2}}{r_{1} r_{12}}\left(\frac{1}{r_{1}} \phi_{n \lambda}\left(\alpha r_{1}\right)\right) g_{k}^{\prime}\left(\gamma r_{12}\right)\right] Y_{l_{1} l_{2} L}\left(\hat{\mathbf{r}}_{1}, \hat{\mathbf{r}}_{2}\right) \\
& +\frac{1}{r_{1}} \phi_{n \lambda}\left(\alpha r_{1}\right) g_{k}\left(\gamma r_{12}\right)\left[w_{c}^{\prime \prime}\left(r_{1}\right)+\frac{2}{r_{1}} w_{c}^{\prime}\left(r_{1}\right)\right] Y_{l_{1} l_{2} L}\left(\hat{\mathbf{r}}_{1}, \hat{\mathbf{r}}_{2}\right)
\end{aligned}
$$

where

$$
\left(\frac{1}{r} \phi_{n \lambda}(\alpha r)\right)^{\prime}=\left(\frac{n+\frac{1}{2} \lambda-1}{r^{2}}-\frac{\alpha}{2 r}\right) \phi_{n \lambda}(\alpha r)-\frac{\sqrt{n(n+\lambda)}}{r^{2}} \phi_{n-1, \lambda}(\alpha r)
$$

and the prime denotes differentiation with respect to the argument, i.e. either $r_{1}$ or $r_{12}$.

The Coulomb interactions become less important as the radius of the confining cavity becomes small (in comparison to the unconfined atomic radius) and the system behaves more and more like electrons in a spherical potential well. Thus, it has been suggested by Aquino et al [17] that trial functions of the form (8), containing decaying exponential terms, may not then be the most appropriate. Consequently, we have also used the solutions of the radial equation

$$
\left(\frac{\mathrm{d}^{2}}{\mathrm{~d} r^{2}}-\frac{l(l+1)}{r^{2}}\right) \chi_{n l}(r)=\epsilon_{n l} \chi_{n l}(r)
$$

to construct basis functions of the form

$$
\phi_{n l}(r)=r^{n+l} \chi_{n l}(r), \quad n=0,1,2, \ldots,
$$

to replace the generalized Laguerre functions $\phi_{n \lambda}\left(\alpha_{v} r_{1}\right)$ and $\phi_{m \mu}\left(\beta_{\nu} r_{2}\right)$ in (7). The solutions to (21) are, of course, simply related to the spherical Bessel functions $j_{l}(r)$; for $l=0$ these solutions are

$$
\chi_{n 0}(r)=\sqrt{\frac{2}{R_{\mathrm{c}}}} \sin \frac{(n+1) \pi r}{R_{\mathrm{c}}}, \quad n=0,1,2, \ldots,
$$

satisfying the Dirichlet boundary conditions, so it is not necessary to introduce cut-off functions here. In our ground-state calculations we employ the functions (23) and evaluate integrals as previously using Gauss-Legendre quadrature. 
Table 1. Convergence of ground-state energies for a helium atom at the centre of an impenetrable spherical cavity of radius $R_{\mathrm{c}}$ as a function of the number of terms in the variational expansion. $N$ is such that $n+m+k \leqslant N$ and $N_{T}$ is the corresponding number of terms in the expansion (6). For each value of $N$ the first row refers to the cut-off function $w_{\mathrm{c}}^{(1)}(r)$ and the second to $w_{\mathrm{c}}^{(3)}(r)$ (see the text).

\begin{tabular}{lrllll}
\hline & & \multicolumn{4}{c}{$R_{\mathrm{c}}$} \\
\cline { 3 - 6 }$N$ & $N_{T}$ & 1.0 & 6.0 & 10.0 & $\infty$ \\
\hline 2 & 7 & 1.0181419 & -2.8989735 & -2.9017479 & -2.9031271 \\
& & 1.0163470 & -2.9017323 & -2.9027158 & \\
3 & 13 & 1.0158358 & -2.9028854 & -2.9034952 & -2.9036364 \\
& & 1.0157856 & -2.9033025 & -2.9035858 & \\
4 & \multirow{2}{*}{22} & 1.0157595 & -2.9035455 & -2.9037042 & -2.9037079 \\
& & 1.0157582 & -2.9036257 & -2.9037107 & \\
5 & \multirow{2}{*}{34} & 1.0157555 & -2.9036526 & -2.9037193 & -2.9037210 \\
& & 1.0157556 & -2.9036716 & -2.9037196 & \\
6 & \multirow{2}{*}{50} & 1.0157551 & -2.9036851 & -2.9037234 & -2.9037237 \\
& & 1.0157551 & -2.9036955 & -2.9037231 & \\
7 & 70 & 1.0157550 & -2.9036922 & -2.9037239 & -2.9037241 \\
& & 1.0157550 & -2.9036938 & -2.9037238 & \\
8 & 95 & 1.0157550 & -2.9036951 & -2.9037242 & -2.9037243 \\
& & 1.0157550 & -2.9036955 & -2.9037241 & \\
\hline
\end{tabular}

\section{Calculations and results}

\subsection{Impenetrable walls}

We have calculated ground-state energies and pressures for a range of confining radii $R_{\mathrm{c}}$. In practice, the sum over $v$ in (6) was reduced to a single term and all terms in the summation satisfying $n+m+k \leqslant 8$ were included, giving a total of 95 terms. When the same limit was imposed on the free-atom calculation the value -2.90372431 resulted for the energy, in excellent agreement with the result -2.903724377 obtained in the extensive and highly accurate calculations of Drake and Yan [24] and Hesse and Baye [12]. The parameters $\alpha$ and $\beta$ were both chosen to have the fixed value 4 , while $\gamma$ was varied to minimize the calculated energy. The optimum value for $\gamma$ depends on the cavity radius. For smaller $R_{\mathrm{c}}$ the energy depends only very slightly on $\gamma$ and $\gamma=0$ which is a suitable choice here. As $R_{\mathrm{c}}$ increased it was found that $\gamma$ initially decreased slowly (to approximately -0.12 at $R_{\mathrm{c}}=3$ ) but then increased to a final value of approximately 0.47 for the unconfined atom. The choice of $\gamma$ is not a critical factor in determining the accuracy of the computed energies; for example, for the free atom $\gamma=0$ gave a ground-state energy of -2.90372409 , as opposed to the final converged value -2.90372431 with $\gamma=0.47$. The number of terms included in the expansion is much more crucial for obtaining an accurate result. This is demonstrated in table 1 which shows how the energy converges as the number of terms in the variational expansions is increased. The table also demonstrates the superiority of the cut-off function $w_{\mathrm{c}}^{(3)}$ over the linear cut-off function $w_{\mathrm{c}}^{(1)}$. As can be observed, however, the differences diminish as the expansion length increases so, again, this choice is not critical, provided a sufficiently large expansion is employed.

The Laguerre basis functions (8) gave lower energies than the basis functions (23) for $R_{\mathrm{c}}$ greater than 0.5 . For values of $R_{\mathrm{c}}$ smaller than 0.5 the Laguerre basis rapidly became unstable 
Table 2. Ground-state energies of a helium atom confined at the centre of an impenetrable spherical cavity of radius $R_{\mathrm{c}}$ in comparison with the results of other workers. The cut-off functions used are $w_{\mathrm{c}}^{(1)}(r)=R_{\mathrm{c}}-r$ and $w_{\mathrm{c}}^{(3)}(r)=\left(1-r / R_{\mathrm{c}}\right) \exp \left(-r / R_{\mathrm{c}}\right)$. The pressure $P$, in atm, is also tabulated.

\begin{tabular}{|c|c|c|c|c|}
\hline$R_{\mathrm{c}}$ & $w_{\mathrm{c}}^{(1)}$ & $w_{\mathrm{c}}^{(3)}$ & Other & $P(\mathrm{~atm})^{\mathrm{a}}$ \\
\hline 0.5 & 22.741303 & 22.741303 & $22.7437^{b}, 22.7413^{c}$ & $0.1167 \times 10^{11}$ \\
\hline 1.0 & 1.015755 & 1.015755 & $\begin{array}{l}1.0176^{\mathrm{b}}, 1.0158^{\mathrm{c}} \\
1.0142 \pm 0.0003^{\mathrm{d}}\end{array}$ & $0.2762 \times 10^{9}$ \\
\hline 2.0 & -2.604038 & -2.604038 & $\begin{array}{l}-2.6026^{b},-2.6040^{c} \\
-2.60403^{\mathrm{e}},-2.6051 \pm 0.0002^{\mathrm{d}}\end{array}$ & $0.4018 \times 10^{7}$ \\
\hline 3.0 & -2.872495 & -2.872495 & $\begin{array}{l}-2.8708^{\mathrm{b}},-2.8725^{\mathrm{c}} \\
-2.87246^{\mathrm{e}},-2.8727 \pm 0.0004^{\mathrm{d}}\end{array}$ & $0.1798 \times 10^{6}$ \\
\hline 4.0 & -2.900485 & -2.900485 & $\begin{array}{l}-2.8988^{\mathrm{b}},-2.9004^{\mathrm{c}} \\
-2.90042^{\mathrm{e}},-2.9003 \pm 0.0006^{\mathrm{d}}\end{array}$ & $0.1074 \times 10^{5}$ \\
\hline 5.0 & -2.903410 & -2.903410 & $\begin{array}{l}-2.9020^{\mathrm{b}},-2.9034^{\mathrm{c}} \\
-2.90337^{\mathrm{e}},-2.9032 \pm 0.0005^{\mathrm{d}}\end{array}$ & $0.6882 \times 10^{3}$ \\
\hline 6.0 & -2.903695 & -2.903696 & $\begin{array}{l}-2.9024^{\mathrm{b}},-2.9037^{\mathrm{c}} \\
-2.90368^{\mathrm{e}},-2.9035 \pm 0.0004^{\mathrm{d}}\end{array}$ & $0.4434 \times 10^{2}$ \\
\hline 8.0 & -2.903724 & -2.903724 & $\begin{array}{l}-2.9025^{\mathrm{b}},-2.9037^{\mathrm{c}} \\
-2.90371^{\mathrm{e}}\end{array}$ & $0.1868 \times 10^{0}$ \\
\hline 10.0 & -2.903724 & -2.903724 & $-2.9037^{\mathrm{c}},-2.90372^{\mathrm{e}}$ & \\
\hline 20.0 & -2.903724 & -2.903724 & & \\
\hline
\end{tabular}

a Present calculation with the cut-off function $w_{\mathrm{c}}^{(3)}(r)$.

b [31].

c [18].

d $[25]$.

e $[26]$.

because of linear dependence problems, so it was not therefore useful for accurate calculations in this range of cavity radii. For very small $R_{\mathrm{c}}$ the Coulomb interactions can be treated as perturbations, resulting in the following expansion for the ground-state energy [27]

$$
E=\frac{E_{0}}{R_{\mathrm{c}}^{2}}+\frac{E_{1}}{R_{\mathrm{c}}}+E_{2}+\cdots
$$

We have $E_{0}=\pi^{2}$ and a straightforward calculation reveals that $E_{1}$ is given by

$$
E_{1}=-4 C i(2 \pi)+2-\frac{2 \operatorname{Si}(2 \pi)-\operatorname{Si}(4 \pi)}{2 \pi}
$$

where $\operatorname{Si}(x)$ and $C i(x)$ are, respectively, the sin and cos integrals [22]. By evaluating $E$ as described in the previous paragraph for a series of small values of $R_{\mathrm{c}}$ we deduce the approximate value $E_{2}=-0.007414$, so that

$$
E=\frac{9.8696044}{R_{\mathrm{c}}^{2}}-\frac{7.9645404}{R_{\mathrm{c}}}-0.007414+\cdots
$$

For $R_{\mathrm{c}}=0.1$, equation (26) gives $E=907.3076$, in comparison to our variational value 907.5625 and its relative accuracy will, of course, increase as $R_{\mathrm{c}}$ takes even smaller values.

In table 2 we compare our energies with those obtained by other workers, and we also include our calculated pressures $P$ evaluated as

$$
P=-\frac{1}{4 \pi R_{\mathrm{c}}^{2}} \frac{\mathrm{d} E}{\mathrm{~d} R_{\mathrm{c}}}
$$


As discussed above, the energies initially fall off very rapidly as $R_{\mathrm{c}}$ increases but subsequently they rapidly approach the asymptotic unconfined-atom value. The pressures initially decrease even faster than the energies for small increasing $R_{\mathrm{c}}$ but then approach zero extremely rapidly as $R_{\mathrm{c}}$ becomes larger. We have used a finite difference scheme to estimate the derivative $\mathrm{d} E / \mathrm{d} R$ and are unable to get reliable values of $P$ for the larger values of $R_{\mathrm{c}}$. We note that our values are in good agreement with those obtained by Aquino et al [17] for $R_{\mathrm{c}} \leqslant 4$ but that the disagreement increases markedly for larger $R_{\mathrm{c}}$. For larger $R_{\mathrm{c}}$, the pressure depends very sensitively on the size of the basis expansion and, in fact, Aquino et al [17] find a difference of approximately a factor of 2 when they double the size of their basis set for $R_{\mathrm{c}}=6$, so their result has obviously not converged for this value of the confining radius. By examining the convergence behaviour as the basis size increases we believe that we have obtained convergence to three significant figures for $R_{\mathrm{c}} \leqslant 6$.

The quantum Monte Carlo calculations of Joslin and Goldman [25] have been referred to in the past as 'exact' and 'nearly exact' (see, for example, $[19,26])$. However, it is clear from table 2 that they are not highly reliable for the smaller values of $R_{\mathrm{c}}$ and, in fact, the more accurate results fall outside Joslin and Goldman's stated error bounds for $R_{\mathrm{c}}<3$, though they do become more reliable as $R_{\mathrm{c}}$ increases, but the error bounds are then not sufficiently tight. In addition, they are computationally very expensive compared to most other calculations. Our ground-state energy results agree, to the four decimal places quoted, with those presented by Flores-Riveros and Rodrigues-Contreras [18] who employed fewer terms in their generalized Hylleraas basis set expansions. There is harmony with the five-decimal-place values quoted by Aquino et al [26], who also employed a generalized Hylleraas expansion, but the present values are lower in all the cases quoted.

We have also computed the atomic ionization radius $I_{R_{\mathrm{c}}}$, that is, the critical radius below which the atom has a higher energy than $\mathrm{He}^{+}$. The required $\mathrm{He}^{+}$energies were obtained from a highly accurate small- $R_{\mathrm{c}}$ expansion in inverse powers of $R_{\mathrm{c}}$ [27]. We find $I_{R_{\mathrm{c}}}=1.38534$, confirming the value 1.385 of Aquino et al [17] and in fair accord with the Sen et al [28] and with Ludẽna [30] who find, respectively, 1.351 and 1.413. In addition, the critical cavity radius at which $E=0$ is found to be $R_{\mathrm{c}}=1.10107$ which again agrees with the result 1.1011 of Aquino et al [17], and may be compared to the values of 1.1013 of Gimarc [29] and 1.1019 of Ludẽna [30].

\subsection{Penetrable walls}

In this section we present some results for the following two penetrable walls:

$$
V_{\mathrm{c}}\left(r_{1}, r_{2}\right)=V_{0}\left[2-\exp \left(-\frac{r_{1}^{2}}{R_{c}^{2}}\right)-\exp \left(-\frac{r_{2}^{2}}{R_{c}^{2}}\right)\right],
$$

and

$$
V_{\mathrm{c}}\left(r_{1}, r_{2}\right)=\frac{1}{2} \omega_{0}^{2}\left(r_{1}^{2}+r_{2}^{2}\right)
$$

which have also been investigated by, respectively, Xie [14] and Sako and Diercksen [15, 16]. The latter authors present results graphically so we are unable to give comparisons with their work. In table 3 we compare our energies with those presented by Xie [14] for the range of $V_{0}$ and $R_{\mathrm{c}}$ values which he considered.

It will be observed that the present energies are, in all cases, lower than those of Xie [14]. For large values of $R_{\mathrm{c}}$ the confining potential can be treated as a small perturbation to the unconfined system. To illustrate this we have also included in table 3 the deviation of $E^{\left(R_{\mathrm{c}}\right)}$ from $E^{(\infty)}$ (i.e. $V_{0}=0$ ) and it can be seen that this is indeed the case. Xie [14] quotes the result $E^{(\infty)}=-2.8999$ for his unconfined ground-state energy but his confined energies for 
Table 3. Ground-state energies of a helium atom confined at the centre of a Gaussian potential well (see equation (28) of text). For each $R_{\mathrm{c}}$ the first row lists the calculated energy while the second gives the energy elevation arising from the confining potential.

\begin{tabular}{|c|c|c|c|c|c|c|}
\hline \multirow[b]{3}{*}{$R_{\mathrm{c}}$} & \multicolumn{6}{|c|}{$V_{0}$} \\
\hline & \multicolumn{2}{|c|}{25} & \multicolumn{2}{|c|}{50} & \multicolumn{2}{|c|}{100} \\
\hline & Present & Xie [14] & Present & Xie [14] & Present & Xie [14] \\
\hline \multirow[t]{2}{*}{1} & 9.0057 & 14.6634 & 15.8461 & 24.7599 & 25.9833 & 39.6224 \\
\hline & 11.9094 & & 18.7499 & & 28.8870 & \\
\hline \multirow[t]{2}{*}{2} & 2.2056 & 5.1046 & 5.2789 & 9.7466 & 9.9194 & 16.6741 \\
\hline & 5.1094 & & 8.1826 & & 12.8231 & \\
\hline \multirow[t]{2}{*}{3} & 0.0586 & 1.9068 & 1.9341 & 4.7940 & 4.8126 & 9.1566 \\
\hline & 2.9623 & & 4.8378 & & 7.7163 & \\
\hline \multirow[t]{2}{*}{4} & -0.937968 & 0.3757 & 0.363210 & 2.4125 & 2.389531 & 5.5249 \\
\hline & 1.965756 & & 3.266934 & & 5.293255 & \\
\hline \multirow[t]{2}{*}{5} & -1.493459 & -0.4799 & -0.523686 & 1.0432 & 1.00694 & $3 . .4227$ \\
\hline & 1.410265 & & 2.380038 & & 3.910418 & \\
\hline \multirow[t]{2}{*}{10} & -2.440318 & -2.0472 & -2.077865 & -1.4363 & -1.474872 & -0.4511 \\
\hline & 0.463406 & & 0.825859 & & 1.428852 & \\
\hline \multirow[t]{2}{*}{15} & -2.675236 & -2.4564 & -2.483038 & -2.1187 & -2.150974 & -1.5555 \\
\hline & 0.228487 & & 0.420689 & & 0.752750 & \\
\hline \multirow[t]{2}{*}{25} & -2.814573 & -2.7109 & -2.733675 & -2.5612 & -2.586843 & -2.2987 \\
\hline & 0.089150 & & 0.170051 & & 0.316880 & \\
\hline \multirow[t]{2}{*}{50} & -2.880334 & -2.8369 & -2.857740 & -2.7929 & -2.814439 & -2.7104 \\
\hline & 0.023391 & & 0.045984 & & 0.089285 & \\
\hline \multirow[t]{2}{*}{100} & -2.897789 & -2.8715 & -2.891912 & -2.8598 & -2.880323 & -2.8369 \\
\hline & 0.005936 & & 0.011812 & & 0.023401 & \\
\hline
\end{tabular}

Table 4. Ground-state energies of a helium atom confined at the centre of a harmonic potential well (see equation (29) of text). The first row lists the energies while the second specifies the perturbation caused by the confining potential.

\begin{tabular}{lrrrrr}
\hline \multicolumn{5}{c}{$\omega_{0}$} \\
\hline \multicolumn{1}{l}{0.01} & \multicolumn{1}{c}{0.05} & \multicolumn{1}{c}{0.1} & \multicolumn{1}{c}{0.25} & \multicolumn{1}{c}{0.5} & \multicolumn{1}{c}{1.0} \\
\hline-2.903605 & -2.900748 & -2.891910 & -2.833069 & -2.648703 & -2.073035 \\
0.000119 & 0.002976 & 0.011814 & 0.070655 & 0.255025 & 0.830689 \\
\hline
\end{tabular}

the largest values of $R_{\mathrm{c}}$ are not consistent with this value; rather, a value in the neighbourhood of -2.883 would be more appropriate. It would appear that, overall, his calculations are not of high accuracy.

Table 4 lists ground-state energies for the harmonic potential (29) for a series of values of the parameter $\omega_{0}$. The deviation of the energy from the unconfined-atom value is also presented and it can be seen that for small $\omega_{0}$ this deviation is approximately linear in $\omega_{0}^{2}$, as can be expected because the confining potential is then a small perturbation of the free atom.

Both potentials (28) and (29) may be used to model the effects of pressure on a helium atom. The softer potential (28) has a finite height $V_{0}$, whereas the harder potential (29) becomes infinite as $r \rightarrow \infty$, so (28) should provide a more realistic model in practice. It also 
has two variable parameters, $V_{0}$ and $R_{\mathrm{c}}$, to choose in fitting experimental data, while (29) has only one, namely $\omega_{0}$.

\section{Conclusions}

We have performed accurate calculations, employing expansions in terms of Hylleraas-type basis functions including the interelectronic distance $r_{12}$, of the ground-state energies of a helium atom confined at the centre of a spherical cavity. Both penetrable and impenetrable cavities have been investigated. For an impenetrable cavity the asymptotic form of the energy as the cavity radius $R_{\mathrm{c}}$ becomes small is given, correct to terms of $O\left(R_{\mathrm{c}}\right)$, by equation (26), and the energies of the atom under pressure have been evaluated for a series of values of $R_{\mathrm{c}}$. The ground-state energies of the atom confined by Gaussian and harmonic penetrable potentials have also been calculated and the results are considered to be the most reliable so far obtained. The numerical procedures employed in the present calculations can easily be applied to any spherical confining potential and to excited states and properties, such as polarizabilities, of helium-like systems. The wavelengths of spectral lines decrease as the pressure increases [32] and it would therefore be of interest to extend the current calculations to excited states.

\section{Acknowledgments}

This work was partially supported by the Chemical Sciences, Geosciences and Biosciences Division of the Office of Basic Energy Sciences, Office of Sciences, US Department of Energy and by the National Science Foundation. We would also like to acknowledge the partial support of the National Science Council of Taiwan (No 97-2112-M-002-003-MY3) and National Taiwan University (No 97R0066).

\section{References}

[1] Michels A, de Boer J and Bijl A 1937 Physica 4981

[2] Sommerfeld A and Welker H 1938 Ann. Phys. 3256

[3] De Groot S R and Ten Seldam C A 1946 Physica 121669

[4] Ley-Koo E and Rubinstein S 1979 J. Chem. Phys. 71351

[5] Fröman P O, Yngve S and Fröman N 1987 J. Math. Phys. 281813

[6] Jaskólski W 1996 Phys. Rep. 2711

[7] Connerade J-P, Dolmatov V K and Lakshmi P A 2000 J. Phys. B: At. Mol. Opt. Phys. 33251

[8] Dolmatov V K, Baltenkov A S, Connerade J-P and Manson S T 2004 Radiat. Phys. Chem. 70417

[9] The Theory of Confined Quantum Systems 2009 Adv. Quantum Chem. 57 Special issue, to be published

[10] Fowler P W 1984 Mol. Phys. 53865

[11] Drake G W F 1988 Can. J. Phys. 66586

[12] Hesse M and Baye D 1999 J. Phys. B.: At. Mol. Opt. Phys. 325605

[13] Gorecki J and Byers Brown W 1988 J. Phys. B: At. Mol. Opt. Phys. 21403

[14] Xie W F 2008 Commun. Theor. Phys. 9491287

[15] Sako T and Diercksen G H F 2003 J. Phys. B: At. Mol. Opt. Phys. 361433

[16] Sako T and Diercksen G H F 2003 J. Phys. B: At. Mol. Opt. Phys. 361681

[17] Aquino N, Riveros A F and Rivas-Silva J F 2003 Phys. Lett. A 307326

[18] Flores-Riveros A and Rodriguez-Contreras A 2008 Phys. Lett. A 3726175

[19] Banerjee A, Kamal C and Chowdhury A 2006 Phys. Lett. A 350121

[20] Gorecki J and Byers Brown W 1987 J. Phys. B: At. Mol. Opt. Phys. 205953

[21] Pekeris C L 1958 Phys. Rev. 1121649

[22] Abramowitz M and Stegun I A 1965 Handbook of Mathematical Functions (New York: Dover)

[23] Drake G W F 1978 Phys. Rev. A 18820

[24] Drake G W F and Yan Z-C 1994 Chem. Phys. Lett. 229486 
[25] Joslin C and Goldman S 1992 J. Phys. B: At. Mol. Opt. Phys. 251965

[26] Aquino N, Garza J, Flores-Riveros A, Rivas-Silva J F and Sen K D 2006 J. Chem. Phys. 124054311

[27] Laughlin C 2009 Adv. Quantum Chem. 57203

[28] Sen K D, Garza J, Vargas R and Aquino N 2002 Phys. Lett. A 295299

[29] Gimarc B M 1967 J. Chem. Phys. 475110

[30] Ludẽna E V 1978 J. Chem. Phys. 691170

[31] Ludẽna E V and Gregori M 1979 J. Chem. Phys. 712235

[32] Varshni Y P 2003 Eur. Phys. J. D 22229 